\title{
A critical analysis and possible modifications of two analytical models for defects sizing using Video Pulse Thermography
}

\author{
by F. Cernuschii ${ }^{1}$ N. Ludwig ${ }^{2}$, P. Teruzzi ${ }^{2}$ and G. Bottazzi ${ }^{3}$
}

${ }^{1}$ ENEL Research, Via Rubattino, 5420134 Milano Italy, E-mail Cernuschi@cram.enel.it

${ }^{2}$ Università degli Studi di Milano, Istituto di Fisica Generale Applicata, Via Celoria, 1620133 Milano Italy, E-mail nicola.ludwig@mi.infn.it

${ }^{3}$ Università degli Studi di Milano, Dipartimento di Fisica, Via Celoria, 1620133 Milano Italy

\begin{abstract}
Two models describing the surface thermal contrast of a sample (containing a sub-surface defect) during a pulse thermography experiment are compared. For both models, multiple reverberations have been taken into account and the effect of the plane wave approximation in treating heat pulse reflections has been analysed by the introduction in the models of a suitable "diaphragm". Moreover for one model an extension to different defects has been proposed. Finally a defect sizing algorithm has been theoretically studied.
\end{abstract}

\section{Introduction}

Nowadays the main topic of research, as far as pulse thermography is concerned, is strongly related to the development of suitable analytical models representing the sample surface temperature distribution vs. time (due to the presence of a sub-surface defect) after a heating pulse. In fact these models allow to simulate the thermal response of a sub-surface defect during a pulse thermography inspection. Starting from theoretical models many inversion algorithms have been proposed in literature for 1D models [1] 2D circular defects [2-6] and 3D defects [7] also using a neural network approach [8]. Good reviews of these models are reported in [9-10].

This work deals mainly with either the extension of 2D models proposed by Almond et al $[2,3]$ and by Crowther et al [5] to more general situations or a sizing algorithm for circular defects.

\section{Theoretical models}

\subsection{Remarks}

The aforementioned two 2D models take the same approach in describing the subsurface defect like a planar scatterer (parallel to the sample surface) of the thermal wave produced by the heat pulse. Both models determine the temperature difference distribution (the thermal contrast) between the region above the defect and a sound zone on the sample surface, using the Green function approach. Almond solves first the problem for single frequency thermal waves and subsequently obtains the transient solution by the Laplace inversion, while Crowther solves directly the equation system in the time domain (i.e. for transient heating). The final solution depends on the scatterer shape function $f$ (i.e. its thermal profile) which is related to the geometrical features of the defect.

Restricting the discussion to the case of circular shaped defects, a flat bottom hole seems to be well described by a "top hat" shape function ( $f=1$ on the defect and 0 outside it) $[2,5]$, while for a circular crack the shape function $f$ is supposed to smoothly vary from 0 (at the crack tip) to 1 (at great distances from the crack tip) [3]. In particular, the equations describing the thermal contrast over the center of a flat bottom hole (located at a depth of $L$ and with a radius $a$ ) for the two models are respectively $[2,5]$ : 


$$
\begin{gathered}
C(\underline{0}, t)=\frac{2 I_{0}}{\varepsilon \sqrt{\pi t}}\left[\exp \left(-\frac{L^{2}}{\alpha t}\right)-\exp \left(-\frac{\left(L+\sqrt{L^{2}+a^{2}}\right)^{2}}{4 \alpha t}\right)\right] \\
C(\underline{0}, t)=\frac{2 I_{0}}{\varepsilon \sqrt{\pi t}}\left[\exp \left(-\frac{L^{2}}{\alpha t}\right)-\frac{1}{2}\left(1+\frac{L}{\sqrt{a^{2}+L^{2}}}\right) \exp \left(-\frac{\left(L+\sqrt{L^{2}+a^{2}}\right)^{2}}{4 \alpha t}\right)\right]
\end{gathered}
$$

where $\alpha=\frac{k}{\rho C}, \varepsilon=\sqrt{k \rho C}$ ( $k$ thermal conductivity, $\rho$ density and $C$ specific heat) and $I_{0}$ are the thermal diffusivity, the thermal effusivity and the energy density of the heating pulse, respectively. Figure 1, reporting the comparison between eq. (1) and (2), shows that the two curves exhibit the same behaviour till their maximum while they differ at longer times.

\subsection{Multiple reverberations, plane waves and the "diaphragm"}

Both eq.(1) and eq.(2) neglect the subsequent reflections of the heat pulse between the sample surface and the defect. In order to take into account in these models the contribution of multiple reverberations it should be considered the superposition of the effects due to an infinite sequence of identical planar circular scatterers placed at uneven multiple depths of the defect depth $\mathrm{L}$ (that is $\pm \mathrm{L}, \pm 3 \mathrm{~L}, \pm 5 \mathrm{~L} \ldots \ldots, \pm(2 \mathrm{n}+1) \mathrm{L}$...). The two solution become [13]:

$$
\begin{aligned}
& C(\underline{0}, t)=\frac{2 I_{0}}{\varepsilon \sqrt{\pi t}}\left\{\sum_{n=1}^{+\infty}\left[\exp \left(-\frac{(n L)^{2}}{\alpha t}\right)-\exp \left(-\frac{(2 n-1) L+\sqrt{L^{2}+a^{2}}}{4 \alpha t}\right)\right]\right\} \\
& C(\underline{0}, t)=\frac{2 I_{0}}{\varepsilon \sqrt{\pi t}}\left\{\sum_{n=1}^{+\infty}\left[\exp \left(-\frac{(n L)^{2}}{\alpha t}\right)-\exp \left(-\frac{L+\sqrt{(2 n-1)^{2} L^{2}+a^{2}}}{4 \alpha t}\right)\right]\right\}
\end{aligned}
$$

Figure 1 shows the thermal contrast $C(\underline{0}, t)$ vs. time as described by eq. (3) and eq. (4). Because of the fast convergence of the two series [13], the sum is restricted to the first 9 terms. The main effects of the multiple reverberations is to increase the amplitude and to extend the time duration of the contrast.

Eq.(3) and eq.(4) have been obtained supposing that each reverberation is the reflection of a plane wave like in the 1D approximation. This is strictly true only for the first term but not for the next ones. A possible solution to eliminate this approximation consists in considering a "diaphragm" (i.e. the characteristic function) on the scatterer plane with an aperture equal to the defect diameter. In this way contributions of the image sources located deeper than $L$ are modified so that it is taken into account that, for subsequent reflections, the waves incident on the scatterer surface can not be assumed plane. Thus the intensity of the heat source shows a smooth profile and also in the case of flat bottomed hole defects, it is no more "top hat" like proposed by both models. This "diaphragm" has been applied only to Crowther model; in particular it has been first determined, using the same Green function, the sum of the contributions of the image sources on the defect plane. The result has been considered different from zero only for points belonging to the scatterer. Finally the effect of this scatterer, with intensity profile computed as just explained, has been evaluated on the sample surface using the same Green function. The final equation is the following: 


$$
C\left(\underline{x \underline{f})}=\frac{-2 Q}{\varepsilon \pi \sqrt{\pi t}}\left\{\begin{array}{l}
\frac{\partial}{\partial}\left[\int_{-\infty}^{+\infty} d x^{\prime} \int_{-\infty}^{+\infty} d y^{\prime}\left\{\exp \left[-(r+L)^{2} / 4 \alpha t\right] / r\right\} \cdot \chi_{\text {defect }}(x, y)\right]+ \\
2 \frac{\partial}{\partial l} \int_{-\infty}^{+\infty} d x^{\prime} \int_{-\infty}^{+\infty} d y^{\prime}\left[\left\{\exp \left[-(r+L)^{2} / 4 \alpha t\right] / r\right\} \sum_{n=1}^{+\infty} \tilde{f}_{n}\left(x^{\prime}, y^{\prime}\right)\right] \chi_{\text {itefect }}(x, y)
\end{array}\right\}\right.
$$

where

$$
f_{n}(x, y)=\frac{\partial}{\partial z}\left[\left.\int_{-\infty}^{+\infty} d x^{\prime} \int_{-\infty}^{+\infty} d y^{\prime} \sum_{n=1}^{+\infty} \frac{\exp \left[-\left(\sqrt{\left(x-x^{\prime}\right)^{2}+\left(y-y^{\prime}\right)^{2}+4 n^{2} L^{2}}+L\right)^{2} / 4 \alpha t\right]}{\sqrt{x^{2}+y^{2}+4 n^{2} L^{2}}}\right|_{z=L} \chi_{\text {deffect }}(x, y)\right]
$$

$\tilde{f}_{n}\left(x^{\prime}, y^{\prime}\right)$ corresponds to $f_{n}(x, y)$ after normalisation, $r=\sqrt{x^{2}+y^{2}+L^{2}}$ and $\chi_{\text {ilefect }}(x, y)$ is the characteristic function.

In figure 1 are reported the curves representing the contrast $C(\underline{0}, t)$ vs. time as described by eq. (4) and as computed applying the procedure herein proposed. Comparing the two curves it is possible to note that they differ only by a small amount. This is true also for the comparison between the spatial profiles (see figure 2) of the thermal contrast. Thus this justifies the approximation, for all reverberations, of plane waves reflection.

\subsection{The non perfect scatterer and two layer samples}

In these models the scatterer has been always supposed to be perfect, i.e. able to completely reflect thermal waves. This corresponds to the hypothesis of a perfectly adiabatic situation. If the nature of the defect does not allow to consider the scatterer as perfect, the partial transmission of the thermal waves through the defect should be taken into account. In the $1 \mathrm{D}$ case, the solutions for homogeneous and semi-infinite two layer samples with partially transmitting defects are respectively $[11,12]$ :

$$
\begin{gathered}
C(0, t)=\frac{2 I_{0}}{\varepsilon \sqrt{\pi t}}\left[\sum_{n=1}^{+\infty} R^{n} \exp \left[-\frac{(n L)^{2}}{\alpha t}\right]\right] \\
C(0, t)=\frac{2 I_{0}}{\varepsilon \sqrt{\pi t}}\left[\sum_{n=1}^{+\infty}\left(R_{1 A}^{n}-R_{12}^{n}\right) \exp \left[-\frac{(n L)^{2}}{\alpha t}\right]\right]
\end{gathered}
$$

where $R=\frac{\varepsilon_{1}-\varepsilon_{2}}{\varepsilon_{1}+\varepsilon_{2}}$ is the thermal wave reflection coefficient at the interface between the material (subscript 1) and the defect (subscript 2). In the case of a two layer sample subscripts $1 \mathrm{~A}$ and 12 refer to reflection coefficient at the interface between the first layer and the defect and between the first layer and the substrate respectively. The extension (based on the analogy with the $1 \mathrm{D}$ solution) to the $2 \mathrm{D}$ solutions for the Crowther model is:

$$
\begin{gathered}
C(\underline{0}, t)=\frac{2 I_{0}}{\varepsilon \sqrt{\pi t}}\left\{\sum_{n=1}^{+\infty} R_{1 A}^{n}\left[\exp \left(-\frac{(n L)^{2}}{\alpha t}\right)-\exp \left(-\frac{L+\sqrt{(2 n-1)^{2} L^{2}+a^{2}}}{4 \alpha t}\right)\right]\right\} \\
C(\underline{0}, t)=\frac{2 I_{0}}{\varepsilon \sqrt{\pi t}}\left\{\sum_{n=1}^{+\infty}\left[\left(R_{1 A}^{n}-R_{12}^{n}\right) \cdot \exp \left(-\frac{(n L)^{2}}{\alpha t}\right)-R_{1 A}^{n} \cdot \exp \left(-\frac{L+\sqrt{(2 n-1)^{2} L^{2}+a^{2}}}{4 \alpha t}\right)\right]\right\}
\end{gathered}
$$

In figure 1 are reported curves describing eq. (8) and eq. (9). 


\subsection{Sizing algorithm}

In single frequency $\omega$ thermal waves experiments, as suggested in literature $[3,14]$, a good measure of the defect diameter (in the case of flat bottom hole) is given by the full width at half maximum (FWHM) of the spatial profile of the thermal contrast. On the contrary for a circular crack (10mm diameter) it resulted that the defect's FWHM is $0.54 \mu$ ( $\mu=\sqrt{2 \alpha / \omega}$ is the thermal diffusion length) smaller than its true defect diameter [3]. Taking into account that a Dirac pulse can be described as the superposition of all frequencies, the following simple equation connecting the measured FWHM at different times with the true diameter in transient thermography experiments was obtained [3]:

$$
F W H M=D_{t r u t}-0.54 \mu_{e f f} \quad\left(\mu_{e f f}=2 \sqrt{\alpha t} \text { and } \mathrm{t} \text { the time }\right)
$$

This is a straight line as a function of the square root of the time. Thus for the correct defect sizing it should be sufficient to consider the value obtained extrapolating eq.(10) (obtained fitting the experimental data) at $t=0$. On the contrary, following this way of thinking, the FWHM(t) of the thermal contrast due to a flat bottom hole should be time independent.

In this work, the FWHM(t) values for a flat bottom hole have been evaluated at different times for the two models with and without the "diaphragm". FWHM has been computed solving numerically the analytical integral equations in $[2,5]$. Moreover, FWHM has been also evaluated using TERMO.HEAT ${ }^{\mathrm{TM}}$ finite differences program both with and without heat losses. The results of FWHM vs. time computations for the different cases are summarised in figure 3 . It is interesting to note that a similar trend (time dependent) is found for each curve. In particular the minimum value of the FWHM corresponds to the time of maximum contrast. These results are in good agreement with the numerical results reported in literature [15]. The comparison between the trends of FWHM as a function of time for models taking into account single or multiple reverberations gives evidence of the weight of multiple reverberation in correctly sizing the defect.

As shown in [16], the experimental results seem to be quite well fitted by a straight line instead of a parabola-like curve. This mismatching between theory and experiments was previously reported by Almond $[3,15]$ in the case of circular crack and is discussed in details for flat bottom hole in [16].

\section{Conclusions}

It has been proposed to extend a $2 \mathrm{D}$ model to the case of multiple reverberations and to describe the defect also like a partially transmitting thermal waves scatterer.

It has been proved that the approximation usually done of supposing for all the reverberations the reflection of plane thermal waves does not affect in a significant way the thermal contrast behaviour. In fact the "diaphragm". introduction modify only by a small amount the spatial and time profile of the thermal contrast.

As far as a defect sizing algorithm is concerned, it has been found that the analytical models give results comparable with the numerical one reported in literature.

\section{Acknowledgments}

Authors want to thank dr. F. Lakestani of Institute for Advanced Material -Joint Research Centre (Ispra -Italy) for his fruitful suggestions.

\section{REFERENCES}

[1] KRAPEZ (J.C.) and BALAGEAS (D.) - Early detection of thermal contrast in pulsed stimulated infrared thermography. - QIRT '94, Proc. of the Eurotherm Seminar 42.1994 p.260-266. 
[2] LAU (S. K.), ALMOND (D. P.) and MILNE (J. M.) - A quantitative analysis of pulsed video thermography. - NDT\&E International, Vol.24 n 41991 p. 195-202.

[3] ALMOND (D. P.) and LAU (S. K.) - Defect sizing by transient thermography. I: an analytical treatment. - J. Phys. D: Appl. Phys. 27, 1994 p.1063-1069.

[4] VAVILOV (V.), MALDAGUE (X.), DUFORT (B.) and ROBITAILLE (J.) - Thermal NDT of carbon epoxy composites: detailed analysis and data processing. - NDT\&E, vol.26 n² 1993 p.85-95.

[5] KRAPEZ (J.), MALDAGUE (X.) and CIELO (P.) - Thermographic NDE: data inversion procedure (part II: 2D analysis and experimental results. - Res. in NDE, vol. $3, n^{\circ} 21991$ p.101-124.

[6] CROWTHER (D. J.), FAVRO (L. D.), KUO (P. K.) and THOMAS (R. L.) - Inverse scattering algorithm applied to infrared thermal wave images. - J. Appl. Phys. $74,(9)$, 1993 p. 5828-5834.

[7] DEGIOVANNI (A.), BENDADA (A.), BATSALE (J. C.) and MAILLET (D.) - Analytical simulation of a multidimensional temperature field produced by planar defects of any shape; application to non destructive testing. - QIRT '94, Proc. of the Eurotherm Seminar 42. 1994 p.253-259.

[8] BISON (P.), BRESSAN (C.), DISARNO (R.), GRINZATO (E.), MARINETTI (S.) and MANDUCHI (G.) - Thermal NDE of delaminations in plastic materials by neural network processing. - QIRT '94, Proc. of the Eurotherm Seminar 42. 1994 p.214-219.

[9] VAVILOV (V.) - TOMOS: Thermal Characterization of Material Defects Using transient Infrared Thermography. - Innovation Inc.1996.

[10] MALDAGUE (X.) - Nondestructive evaluation of materials by infrared thermography. Springer Verlag London 1993.

[11] CARSLAW (H. S.) and JAEGER (T. L.) - Conduction of Heat in Solids Oxford University Press $2^{\text {nd }}$ Edition 1959.

[12] CERNUSCHI (F.), COLOMBO (E.), RUSSO (V.), SALERNO (A.), GHIA (S.) and MARCHESI (R.) - Ceramic coating non destructive quality control by using video pulse thermography.- Tranchina (B.S.) and. Bellosi (A.) eds. 4th EURO-CERAMICS, . - Coatings and Joinings Vol. 9, 1995, p.419-428.

[13] CERNUSCHI (F.), GHIA (S.), LUDWIG (N.), RUSSO (A.) and TERUZZI (P.) - Una nuova metodologia per il dimensionamento dei difetti sub-superficiali con la termografia impulsata. - Proc. $9^{\circ}$ Congresso Nazionale AIPnD 1997, p.799-813.

[14] WETSEL (G. C.) and MCDONALD (F. A.) - Resolution and definition in photohermal imaging. - J. Appl. Phys. 56, 1984 p.3081-3085.

[15] SAINTEY (M. B.) and ALMOND (D. P.) - Defect sizing by transient thermography II: a numerical treatment. - J. Phys. D: Appl. Phys., 281995 p. 2539-2546.

[16] CERNUSCHI (F.) LUDWIG (N.) and TERUZZI (P.) - Statistical based procedure for defect sizing and experimental evaluation of the convective effects using video pulse thermography. - Presented at QIRT'98, Eurotherm Seminar n60 1998. 


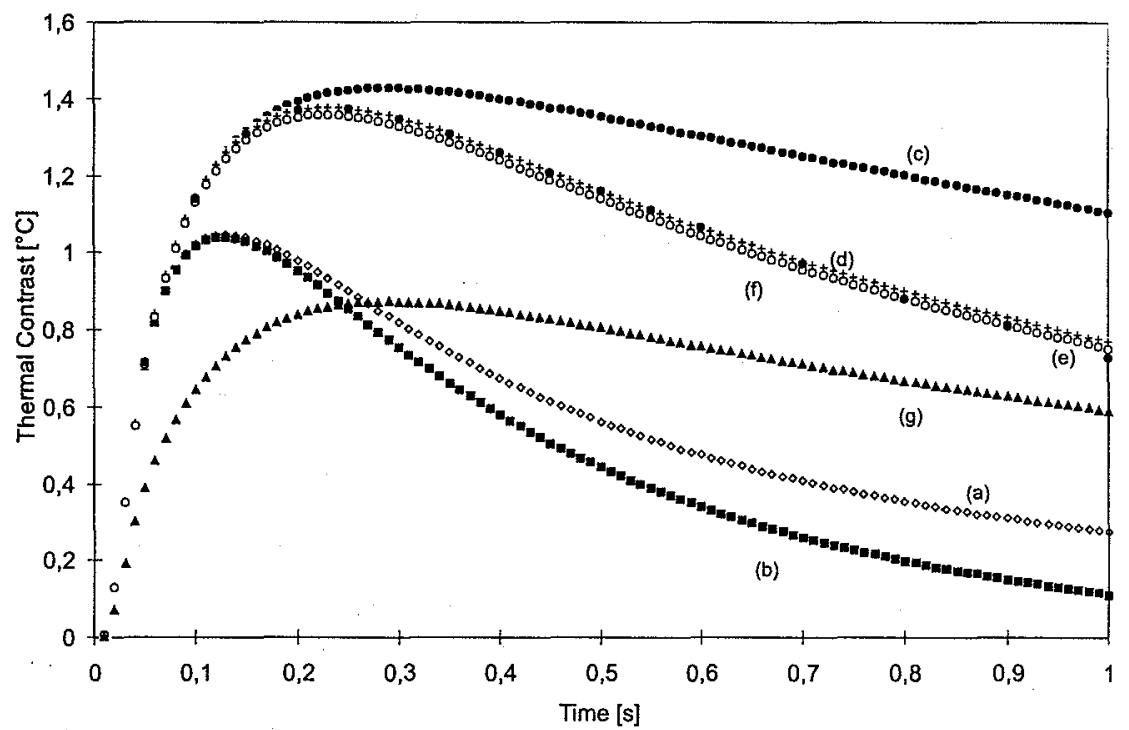

Fig. 1. Thermal contrast $C(0, t)$ vs. time as described by (a) eq.(1), (b) eq.(2),(c) eq.(3), (d) eq.(4), (e) eq.(5), (f) eq.(8) and (g) eq. (9). Curves (a)-(e) were evaluated for a mild steel $\left(\alpha=0.14 * 10^{-4} \mathrm{~m}^{2} / \mathrm{s}\right.$ and $\left.\varepsilon=17100 \mathrm{~J} / \mathrm{m}^{2} \mathrm{~s}^{1 / 2} \mathrm{~K}\right)$ sample containing a flat bottom hole at a depth $L=1 \mathrm{~mm}$, with a radius $a=5 \mathrm{~mm}$ and for heating energy density $l_{0}=10000 \mathrm{~J} / \mathrm{m}^{2}$.

Curve $(\mathrm{g})$ was evaluated for mild steel first layer and a nickel alloy substrate $\left(\alpha=0.03 * 10^{-4} \mathrm{~m}^{2} / \mathrm{s}\right.$ and $\left.\varepsilon=6468 \mathrm{~J} / \mathrm{m}^{2} \mathrm{~s}^{1 / 2} \mathrm{~K}\right)$. In $(\mathrm{f})$ and $(\mathrm{g})$ the defect is considered as air $\left(\alpha=0.2 * 10^{-4} \mathrm{~m}^{2} / \mathrm{s}\right.$ and $\left.\varepsilon=5.5 \mathrm{~J} / \mathrm{m}^{2} \mathrm{~s}^{1 / 2} \mathrm{~K}\right)$

Fauro Model with mewerberations

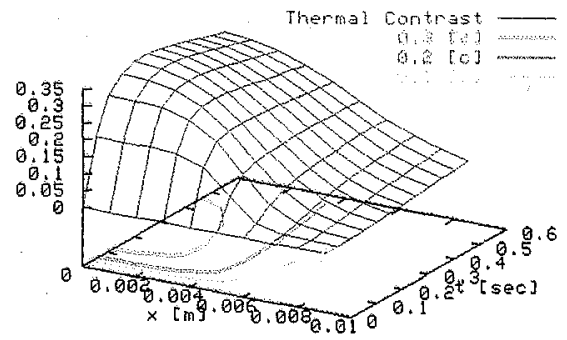

Dlaphragm Model with reverberations

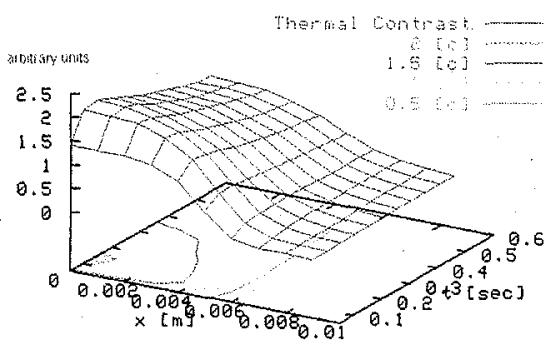

Fig. 2. Thermal contrast $C(x, t)$ vs. spatial position at different times as described by Crowther model (a) with and (b) without "diaphragm". Curves were evaluated using the same parameters of fig. 1 
http://dx.doi.org/10.21611/qirt.1998.033

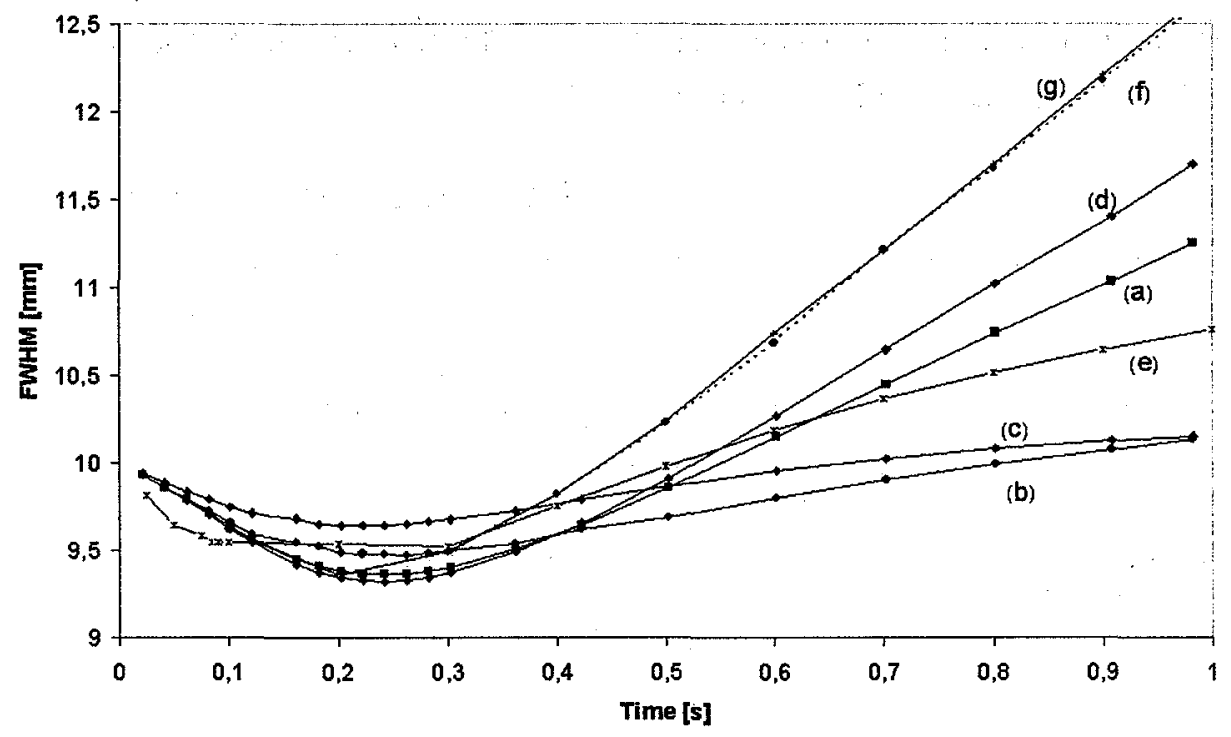

Fig. 3. FWHM of the thermal contrast $C(x, t)$ vs. time. Curves were evaluated using the same parameters of fig. 1. In particular Almond's model for (a) one reverberation, (b) multiple reverberations, Crowther's model for (c) one reverberation, (d) multiple reverberations, (e) "diaphragm" model and TERMO.HEAT $T^{\mathrm{TM}}$ simulation (f) without heat losses, $(g)$ with heat losses $\left(h=100 W / m^{2} K\right)$ 